\title{
CORRECTION DE DISPERSION POUR L'ANALYSE DES PETITES DÉFORMATIONS AUX BARRES DE HOPKINSON
}

\author{
G. GARY* , J.R. KLEPACZKO** et H. ZHAO* \\ *Laboratoire de Mécanique des Solides, École Polytechnique, \\ F-91128 Palaiseau Cedex, France \\ ** Université de Metz, LPMM-CNRS, Ile du Saulcy, F-57045 Metz \\ Cedex, France
}

Résumé. Nous présentons une méthode d'analyse de l'essai SHPB qui permet d'accéder au comportement de l'échantillon dans la zone des faibles déformations de l'ordre de $10^{-3}$. Validée par des simulations numériques, cette méthode est fondée sur l'utilisation de techniques opérationnelles sur ordinateur compatible IBM-PC. Elle prend en compte la correction de dispersion des ondes dans des barres constituées d'un matériau vicoélastique linéaire quelconque. L'équation de dispersion de Pochhammer et Chree est généralisée à ce type de matériau, puis résolue.

Abstract. A method for the analysis of SHPB test is presented which allows for determination of the behaviour of a specimen in the range of small strains in the order of $10^{-3}$. This method is validated by numerical simulations. It is tested on a IBM-PC computer. The method takes into account wave dispersion effects in bars of any linear viscoelastic material. The Pochhammer Chree dispersion equation is derived and solved for this kind of material.

\section{INTRODUCTION}

En général, il est admis que la relation contrainte-déformation d'un matériau, établie à partir d'un essai aux barres de Hopkinson ou SHPB (Split Hopkinson Pressure Bar, "Kolsky apparatus"), ne permet pas de mesurer les caractéristiques élastiques de ce matériau ou, dit autrement, que la zone des déformations inférieures à $1 \%$ n'est pas accessible par cet essai $/ 1,2,3 /$. Nous montrons que cette limitation n'est pas inhérente à la technique de mesure SHPB et nous proposons une méthode de dépouillement complète, qui prend notamment en compte les effets de dispersion dans les barres et qui permet d'accéder aux petites déformations (inférieures à 1\%) de l'échantillon. Cette méthode est mise en oeuvre par un logiciel implanté sur un ordinateur du type compatible IBM-PC-AT de sorte qu'elle est utilisable en temps quasi réel et en ambiance de laboratoire d'essais. Nous servons ainsi au passage un de nos objectifs qui est de mettre à la portée des laboratoires industriels une méthode efficace et optimale de dépouillement de l'essai SHPB qui soit simple d'emploi, rapide et complète.

\section{POSITION DU PROBLEME}

En se restreignant à l'hypothèse du comportement uniaxial en contrainte de l'échantillon, on montre, à partir de simulations, que la loi de comportement peut etre obtenue, même dans cette zone, à partir de la connaissance exacte des trois ondes "incidente", "réfléchie" et "transmise", par la simple utilisation d'une méthode de dépouillement directe.

Une telle méthode est fondée sur la connaissance de la propagation des ondes élastiques dans les barres (fig. 1). Les deformations mesurées sur les barres $\epsilon_{\mathrm{i}}, \epsilon_{\mathrm{r}}$, et $\epsilon_{\mathrm{t}}$ correspondant aux ondes incidente, refléchie et transmise, qui interviennent dans les formules de cette figure, sont associées aux extrémités des barres, c'est à dire aux interfaces barre-échantillon alors qu'elles sont mesurées par des jauges collées à une certaine distance. On doit donc procéder à un transport fictif des trois ondes de base, en avançant dans le temps pour l'onde incidente et en remontant dans le temps pour les ondes réfléchie et transmise.

Deux difficultés sont liées à ce transport. La première est de déterminer les origines des trois ondes pour qu'elles correspondent à un même moment de l'essai qui est -par exemple- celui où l'onde incidente 
atteint la face entrante de l'échantillon (difficulté D1). La deuxième tient au caractère tridimensionnel et parfois viscoćlastique des barres qui introduit une dispersion dans la propagation des ondes; dispersion géométrique et dispersion due à l'éventuel caractère viscoélastique du matériau (difficulté D2) $/ 3,4 /$.

Deformation

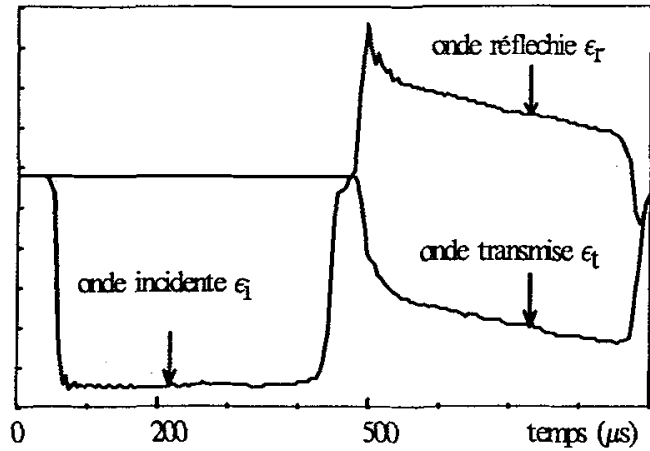

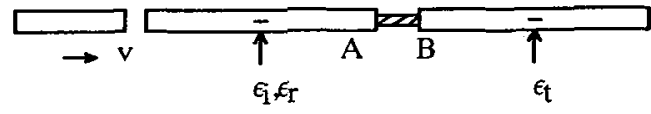

$\mathrm{F}_{\mathrm{A}}=\mathrm{E}_{\mathrm{b}} \mathrm{S}_{\mathrm{b}}\left(\epsilon_{\mathrm{i}}+\epsilon_{\mathrm{r}}\right)$

$\mathrm{V}_{\mathrm{A}}=\mathrm{C}_{\mathrm{Ob}}\left(\epsilon_{\mathrm{i}}-\epsilon_{\mathrm{r}}\right)$

méthode "conplète"

$\sigma=\mathrm{E}_{\mathrm{b}} \mathrm{S}_{\mathrm{b}}\left(\epsilon_{\mathrm{i}}+\epsilon_{\mathrm{r}}+\epsilon_{\mathrm{t}}\right) / 2 \mathrm{~S}_{\mathrm{e}}$ $\dot{\varepsilon}=\mathrm{C}_{0 \mathrm{~b}}\left(\epsilon_{\mathrm{i}}-\varepsilon_{\mathrm{r}}-\varepsilon_{\mathrm{t}}\right) / \mathrm{l}_{\mathrm{e}}$

Ef: Module d'Yamg des barres $\mathrm{S}_{\mathrm{b}}$ : Section des banes Cob: Célérité dans les barres

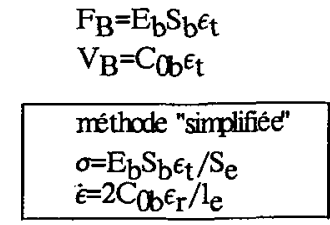

le: Longuar đédhantillon

$\mathrm{S}_{\mathrm{e}}$ : Section déchantillon

\section{fig.1 Méthodes "complète"( 3 ondes) et "simplifiée" ( 2 ondes) de dépouillement de l'essai SHPB}

D'autres dificultés sont liées à l'essai de comportement lui même (nous nous limitons ici à l'essai de compression). Les méthodes classiques de dépouillement présentées sur la figure 1 négligent en effet le caractère transitoire du chargement puisqu'elles supposent l'homogénéité des champs de déformation et de contrainte par rapport à la direction du chargement (difficulté D3). On trouve aussi bien sûr toutes les difficultés tenant au caractère unidimensionnel des mesures effectuées sur un échantillon tridimensionnel./5,6/ Ces difficultés ont été étudiées par de nombreux auteurs qui ont proposé des méthodes de correction afin de prendre en compte les frottements aux interfaces barre-échantilon et les effets d'inertie radiaux./7/ Il n'est bien entendu pas dans notre intention de minimiser ces dernières difficultés mais, dans le but de clarifier notre approche, nous nous limiterons volontairement ici au cas du comportement uniaxial en contrainte de l'échantillon, ce qui, en conséquence, nous permettra de concentrer nos efforts sur les difficultés propres à l'appareil de mesure SHPB dans le domaine des petites déformations.

\section{SIMULATION D'UN ESSAI DE COMPRESSION}

Afin de tester les techniques de dépouillement de l'essai SHPB, nous simulons un essai de compression réalisé sur un matériau dont la loi de comportement est donnée, et donc connue par avance. L'onde incidente étant également donnée, on calcule ainsi les ondes transmise et réfléchie. On dépouille alors l'ensemble des trois ondes comme si elles résultaient d'un essai réel.

Afin de nous situer dans un cadre réaliste, les données d'entrée de la simulation seront inspirées d'une situation réelle : caractéristiques géométriques (longueur, diamètre) et mécaniques (module d'Young) réelles des barres; onde incidente de forme trapézoidale; géométrie de l'échantillon et propriétés mécaniques du matériau modèle inspirées d'un matériau réel de type métallique (laiton par exemple). La réponse de l'échantillon est donc supposée en contrainte uniaxiale et évidemment transitoire.

Différents modèles de calcul ont été développés, adaptés à chaque type de loi de comportement. Conformément à notre objectif de développer des méthodes facilement utilisables, un effort particulier a été apporté à la recherche de rapidité des algorithmes afin de pouvoir ultérieurement les utiliser efficacement dans des techniques de calcul inverse, implantées sur ordinateur compatible PC.

Dans un but de généralité, en particulier pour être capable de décrire la réponse de matériaux présentant de l'écrouissage négatif (matériaux de type rocheux), nous utilisons, pour décrire le matériau de l'échantillon une formulation de la loi de comportement du type Malvern-Sokolowsky /8,9/. La déformation est décomposée en une partie élastique et une partie non élastique et on suppose alors que l'incrément de la déformation non élastique ne dépend que de l'êtat actuel de la contrainte et de la déformation totale. La loi de comportement s'écrit :

$$
\frac{\partial \epsilon}{\partial \mathrm{t}}=\frac{1}{\mathrm{E}} \frac{\partial \sigma}{\partial \mathrm{t}}+\mathrm{g}(\sigma, \epsilon)
$$


On peut interpréter physiquement cette loi en considérant que les ondes se propagent avec une célérité élastique $\mathrm{C}_{0}$ constante et qu'il y a relaxation de la contrainte en cours de propagation. On peut montrer que le modèle rhéologique élasto-visco-plastique à écrouissage linéaire (voir fig.2) peut être correctement décrit par une représentation de ce type; dans ce cas on a:

$$
g(\sigma, \epsilon)=\left[\left(1+E_{t} / E\right) \sigma-E_{t} \epsilon-\sigma_{y}\right] / \eta
$$

avec : $E$ module d'Young, $E_{t}$ module d'écrouissage, $\eta$ coefficeint de viscosité, $\sigma_{\mathrm{y}}$ limite d'élasticité Contrainte (MPa)

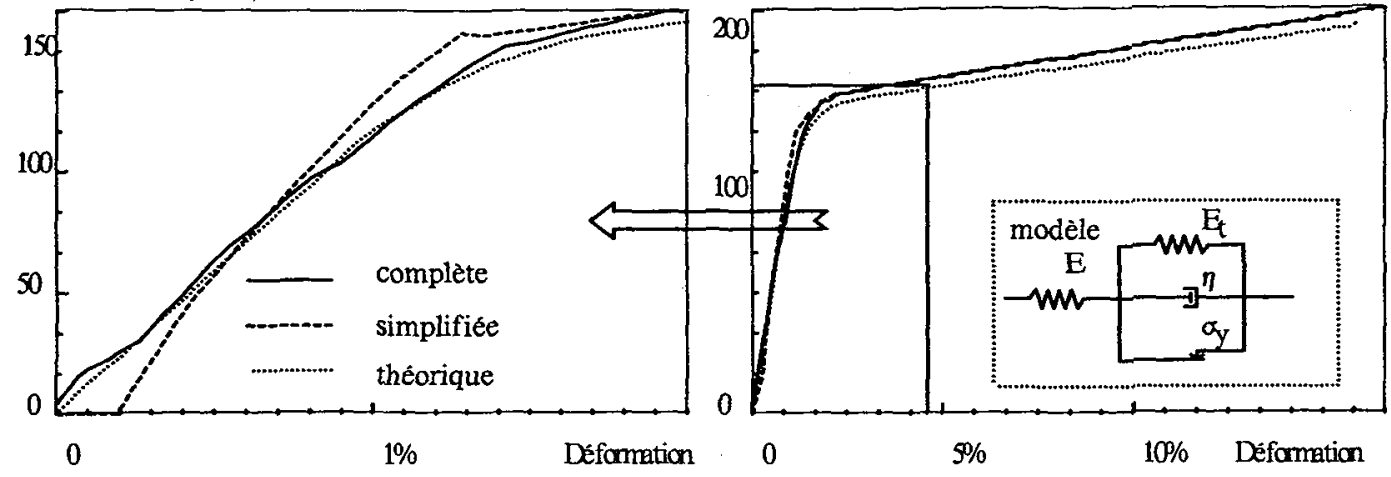

fig.2 Simulation élasto-visco-plastique et validation des méthodes de depouillement

C'est un tel modèle à quatre paramètres que nous avons d'ailleurs utilisé pour les simulations présentées ici (eq. 2). Son utilisation et la prise en compte des conditions aux limites de l'essai conduit à un système d'équations aux dérivées partielles d'ordre 1 qui s'intègre par la méthode des caractéristiques. Nous calculons donc les ondes réfléchie et transmise correspondant à l'essai simulé.

L'ensemble est dépouillé avec le logiciel DAVID /10/ qui utilise l'une ou l'autre des deux méthodes précisées sur la figure 1 . Nous présentons sur la figure 2 l'exemple défini comme suit : barres de Hopkinson en acier de module d'Young $2.10^{5} \mathrm{MPa}$; de masse volumique $7850 \mathrm{~kg} / \mathrm{m}^{3}$; diamètre $16.5 \mathrm{~mm}$; célérité des ondes elastiques $4810 \mathrm{~m} / \mathrm{s}$; impacteur identique aux barres de longueur $0.9 \mathrm{~m}$. ; vitesse du tir $14 \mathrm{~m} / \mathrm{s}$ ; éprouvette : diamètre $0.016 \mathrm{~m}$; longueur $0.01 \mathrm{~m}$; masse volumique du matériau $8000 \mathrm{~kg} / \mathrm{m}^{3}$; paramètres du modèle (eq.2) : $E=1.110^{4} \mathrm{MPa} ; E_{\mathrm{t}}=4.110^{2} \mathrm{MPa}$ (soit $3.6 \%$ de $\mathrm{E}$ ); $\sigma_{\mathrm{y}}=100 \mathrm{MPa} ; \eta=10^{5} \mathrm{~Pa} . \mathrm{s}$

Deformation

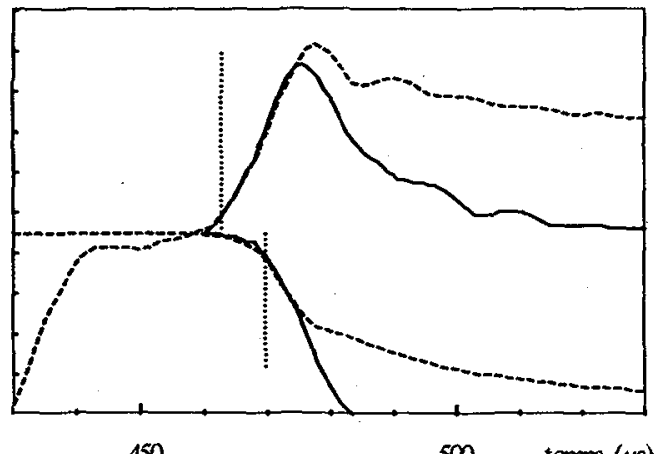

position des origines d'après la simulation

-....... onde réelle mesurée

_meilleure" réponse elastique simulée

$\left(\mathrm{E}=1.1 \quad 10^{5} \mathrm{M} \mathrm{Pa}\right)$

"Zoom" sur le début des ondes transmise et réfléchie

fig.3 Choix des origines des ondes par simulation élastique

On constate sur ia figure 2, que la méthode complète (calcul avec 3 ondes) donne un meilleur résultat que la méthode simplifiée (calcul avec 2 ondes). On constate surtout que cette méthode donne un résultat proche de la loi idêale, notamment pour la zone élastique. Ce bon résultat n'est pas couramment admis. Nous l'obtenons ici, car les difficultés liées au transport des ondes, (dificultés D1 et D2), n'existent pas dans le cas d'une simulation. Il montre également que l'hypothèse d'homogénéité axiale n'est pas très forte 
pour ce genre de matériaux (difficulté D3 peu importante).

C'est pourquoi nous sommes fondés à incorporer à notre technique de dépouillement des essais réels une méthode permettant un calage relatif précis des trois ondes de base (D1) et prenant en compte la correction de dispersion dans les barres (D2).

\section{RECALAGE DES ONDES.}

La technique que nous proposons est fondée sur l'hypothèse que le matériau étudié a, pour les déformations faibles, un comportement élastique, hypothèse raisonnable pour la plupart des matériaux. Le début de l'onde incidente étant défini, on calcule les ondes réfléchie et transmise que l'on obtiendrait avec un matériau élastique de module connu. Ce calcul est effectué en temps réel. Il est introduit dans le logiciel de dépouillement DAVID et permet, de manière interactive, de trouver la meilleure valeur du module qui fait coincider le début des ondes réelles avec le début des ondes élastiques simulées. La position du zéro des ondes réfléchie et transmise en est alors déduite de façon systématique, fig. 3

\section{CORRECTIONS DE DISPERSION.}

Comme nous voulons que la correction de dispersion soit opérationnelle dans un logiciel portable sur un micro ordinateur compatible PC (en l'occurence DAVID), il n'est pas possible d'effectuer un calcul tridimensionnel. Nous utilisons donc d'une approche basée sur l'analyse développée par Pochhammer et Chree, puis par Davies /4,11,12/. Cette approche théorique traite le cas de la propagation d'ondes stationnaires dans une barre infinie et n'est donc pas strictement correcte dans le cas des barres de Hopkinson. Cette hypothèse est admise par de nombreux auteurs $/ 13 /$. Elle donne des résultats très satisfaisants dans le cas des barres élastiques comme nous l'avons vérifié et comme cela est montré également dans $/ 2,10 /$. Par contre, si le matériau constituant les barres manifeste des effets viscoélastiques, cette correction géométrique n'est plus suffisante, fig. 4. Or on sait bien qu'il n'existe pas de matériau élastique ayant une impédance assez faible pour permettre l'étude de certains matériaux (bois légers, caoutchoucs, mousses de polyuréthane) par le système SHPB et on a fréquemment recours à des barres en PMMA, matériau ayant des caractéristiques élastiques correctes mais présentant malheureusement des effets viscoélastiques non négligeables. Il nous a donc paru essentiel d'introduire une correction prenant à la fois en compte l'effet géométrique et l'effet viscoélastique.

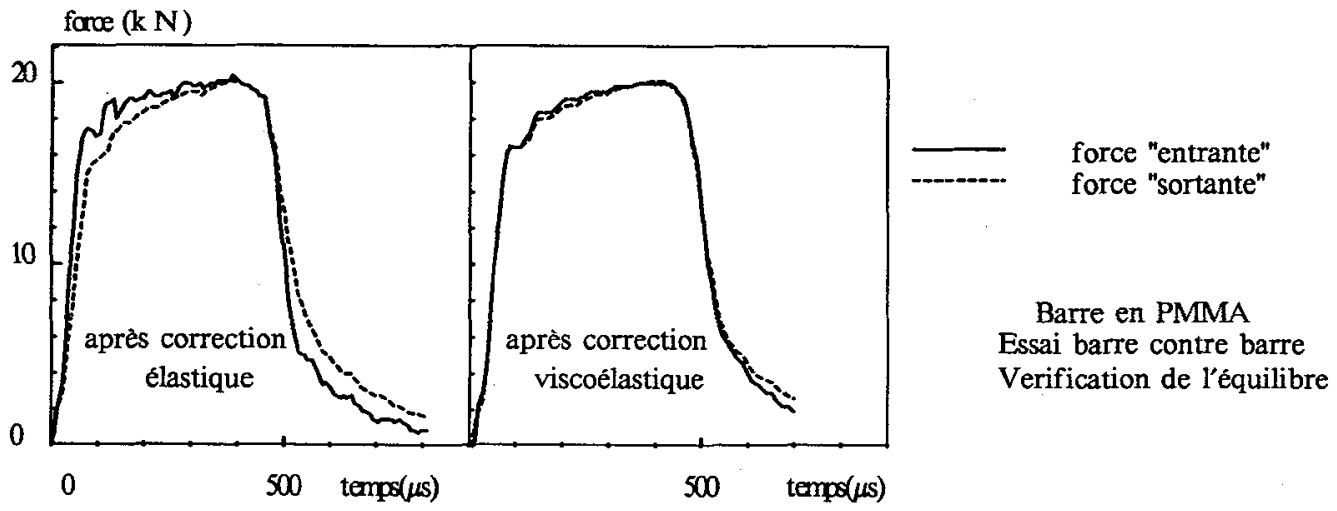

fig.4 Nécessité de la correction "Pochhammer-Chree viscoélastique couplée" pour une barre viscoélastique

Il aurait été simple de traiter séparément les effets géométriques élastiques et les effets visqueux, mais aucune justification théorique ne permet de supposer que ces deux effets sont découplés. Nous avons donc résolu le problème couplé. La méthode utilisée est inspirée de celle utilisée pour résoudre le problème élastique telle que présentée dans $/ 4,11,15 /$ et plus récemment rappelée dans $/ 16 /$.

\subsection{APPROCHE THEORIQUE. ONDES DANS UN MILIEU VISCOELASTIQUE LINEARE INFINI}

Les équations générales de la dynamique s'écrivent :

$\operatorname{div} \sigma+f=\rho \frac{\partial^{2} u}{\partial t^{2}}$ 
où $\mathbf{u}$ est le vecteur déplacement, $f$ représente les forces de volume, $\rho$ masse volumique du matériau.

Nous nous plaçons dans l'hypothèse des petites transformations où notamment :

$\epsilon=1 / 2\left[\operatorname{grad} u+\operatorname{grad}^{t} u\right]$

La loi de comportement visco-élastique linéaire isotrope s'écrit généralement sous forme intégrale de la façon suivante :

$$
\sigma(t)=\int_{-\infty}^{t}\left[\lambda(t-\tau) \operatorname{tr}\left(\frac{\partial \epsilon(\tau)}{\partial \tau}\right) 1+2 \mu(t-\tau) \frac{\partial \epsilon(\tau)}{\partial \tau}\right] d \tau
$$

On suppose que la solution $\mathbf{u}$ recherchée est une combinaison d'ondes harmoniques et on s'intéresse dans ce qui suit au cas d'une onde harmonique élémentaire de pulsation $\omega$.

$$
\mathbf{u}=\mathbf{u}^{*} \mathrm{e}^{-i \omega t}
$$

On introduit les contraintes et les déformations associées, où

$\sigma^{*}$ et $\epsilon^{*}$ sont complexes.

$$
\sigma=\sigma^{*} \mathrm{e}^{-i \omega t} \text { et } \epsilon=\epsilon^{*} \mathrm{e}^{-i \omega t}
$$

Les équations (3),(4) et (5) s'écrivent alors

$\operatorname{div} \sigma^{*}+\mathbf{f}^{*}=\rho \omega^{2} \mathbf{u}^{*}$

$2 \epsilon^{*}=\operatorname{grad} u^{*}+\operatorname{grad}^{t} u^{*}$

$\sigma^{*}=\lambda^{*}(\omega) \operatorname{tr}\left(\epsilon^{*}\right) 1+2 \mu^{*}(\omega) \epsilon^{*}$

En l'absence de forces volumiques nous en déduisons l'équation du mouvement suivante

$\mu^{*}(\omega) \Delta \mathbf{u}^{*}+\left[\lambda^{*}(\omega)+\mu^{*}(\omega)\right] \operatorname{grad} \operatorname{div} \mathbf{u}^{*}=-\rho \omega^{2} \mathbf{u}^{*}$

Nous nous intéressons maintenant aux solutions $\mathbf{u}^{*}$ de la forme suivante

$\mathbf{u}^{*}=\operatorname{grad} \phi^{*}+\operatorname{rot} \mathbf{H}^{*}$

où $\phi^{*}$ est un fonction scalaire et $\mathbf{H}^{*}$ un vecteur. L'équation (12) s'écrit alors :

$\mu^{*}(\omega) \Delta\left(\operatorname{grad} \phi^{*}+\operatorname{rot} H^{*}\right)+\left[\lambda^{*}(\omega)+\mu^{*}(\omega)\right] \operatorname{grad} \operatorname{div}\left(\operatorname{grad} \phi^{*}+\operatorname{rot} H^{*}\right)=-p \omega^{2}\left(\operatorname{grad} \phi^{*}+\operatorname{rot} H^{*}\right)$

Comme $\operatorname{div} \operatorname{grad}(\phi)=\Delta \phi$ et $\operatorname{div} \operatorname{rot} \mathbf{H}=0$ l'équation 14 s'écrit encore :

$\operatorname{grad}\left[\left(\lambda^{*}(\omega)+2 \mu^{*}(\omega)\right) \Delta \phi^{*}+\rho \omega^{2} \phi^{*}\right]+\operatorname{rot}\left[\mu^{*}(\omega) \Delta \mathbf{H}^{*}+\rho \omega^{2} \mathbf{H}^{*}\right]=0$

On en déduit que $\phi^{*}$ et $+H^{*}$ doivent vérifier les équations classiques suivantes:

$$
\begin{aligned}
\Delta \phi^{*}+\omega^{2} /\left(c_{1}^{*}\right)^{2} \phi^{*} & =0 & \text { avec } & c_{1}^{*} & =\left[\left(\lambda^{*}(\omega)+\mu^{*}(\omega)\right) / \rho\right]^{1 / 2} \\
\Delta \mathbf{H}^{*}+\omega^{2} /\left(c_{2}^{*}\right)^{2} \mathbf{H}^{*} & =0 & \text { avec } & c_{2}^{*} & =\left[\mu^{*}(\omega) / \rho\right]^{1 / 2}
\end{aligned}
$$

\subsection{CAS D'UN CYLINDRE INFINI}

En s'inspirant de l'approche de Pochhammer et Chree, on se place dans un repère cylindrique d'axe z (avec les variables $\mathrm{r}$ et $\theta$ ). On cherche ensuite des solutions pour $\phi^{*}$ et $\mathrm{H}^{*}$ de forme séparable, où $\mathrm{f}, \mathrm{g}, \mathrm{h}_{\mathrm{X}}$ et $\boldsymbol{\theta}_{\mathrm{X}}$ sont des fonctions complexes :

$$
\begin{aligned}
& \phi^{*}=\mathrm{f}(\mathrm{r}) \cdot \mathrm{g}(\theta) \cdot \mathrm{e}^{\mathrm{i} \xi \mathrm{z}} \\
& \mathrm{H}^{*}=\left[\mathrm{h}_{1}(\mathrm{r}) \cdot \theta_{1}(\theta) \cdot \mathrm{e}^{\mathrm{i} \xi \mathrm{z}}\right] \mathrm{r}+\left[\left[\mathrm{h}_{2}(\mathrm{r}) \cdot \theta_{2}(\theta) \cdot \mathrm{e}^{\mathrm{i} \xi \mathrm{z}}\right] \theta+\left[\mathrm{h}_{3}(\mathrm{r}) \cdot \theta_{3}(\theta) \cdot \mathrm{e}^{\mathrm{i} \xi \mathrm{z}}\right] \mathrm{z}\right.
\end{aligned}
$$


On reporte ensuite ces expressions de $\phi^{*}$ et de $\mathbf{H}^{*}$ dans les équations (16) et (17). vantes.

Dans le cas de $\phi^{*}$, on en déduat les deux équations différentielles ordinaires à valeurs complexes sui-

$$
\begin{aligned}
& \frac{\mathrm{d}^{2} \mathrm{f}}{\mathrm{d} \mathrm{r}^{2}}+\frac{1}{\mathrm{r}} \frac{\mathrm{df}}{\mathrm{dr}}+\left(\frac{\omega^{2}}{c_{\mathrm{t}}{ }^{2}}-\xi^{2}\right) f-\frac{\mathrm{n}^{2}}{\mathrm{r}^{2}} \mathrm{f}=0 \\
& \frac{\mathrm{d}^{2} \mathrm{~g}}{\mathrm{~d} \theta^{2}}+\mathrm{n}^{2} \mathrm{~g}(\theta)=0
\end{aligned}
$$

Les solutions de l'équation (21) sont des fonctions sinus et cosinus de $\mathrm{n} \theta$ où $\mathrm{n}$ est un entier positif ou nul. L'équation (20) est une équation de Bessel dont la solution est donc une fonction de Bessel :

$$
\phi=\left[a_{1} \cos (n \theta)+a_{2} \sin (n \theta)\right] \text { Jn }(\alpha r) \text {, avec : } \alpha=\frac{\omega^{2}}{c_{1}{ }^{2}}-\xi^{2}
$$

Dans le cas de $H^{*}$, on procède de la même façon. $\beta$ a la même expression que $\alpha$ avec $C_{2}$ a la place de $\mathrm{C}_{1}$. On est donc en mesure de calculer les champs de contrainte et de déplacement. En prenant en compte les conditions aux limites pour une barre infinie de rayon a et dans le cas des ondes harmoniques longitudinales on retrouve l'équation de fréquence classique établie dans le cas élastique par Pochhammer et Chree. Ici bien sar la solution est à valeurs complexes.

$$
(2 \alpha / a) \cdot\left(\alpha^{2}+\xi^{2}\right) . \mathrm{J}_{1}(\alpha \mathrm{a}) \mathrm{J}_{1}(\beta \mathrm{a})-\left(\beta^{2}-\xi^{2}\right)^{2} . \mathrm{J}_{0}(\alpha \mathrm{a}) \cdot \mathrm{J}_{1}(\beta \mathrm{a})-4 \cdot \xi^{2} \cdot \alpha \cdot \beta . \mathrm{J}_{1}(\alpha \mathrm{a}) . \mathrm{J}_{0}(\beta \mathrm{a})=0
$$

Rappellons que $\xi$ représente un déphasage "complexe", la partie réelle représentant le déphasage et la partie imaginaire l'amortissement. On retrouvera donc d'une part un résultat comparable à celui de l'élasticité exprimant une relation entre fréquence et vitesse de phase (qu'on ne pourra toutefois pas représenter sous forme réduite comme en élasticité ) et d'autre part une relation entre fréquence et amortissement.

\section{RFSULTATS ET APPLICATION AU SYSTEME SHPB}

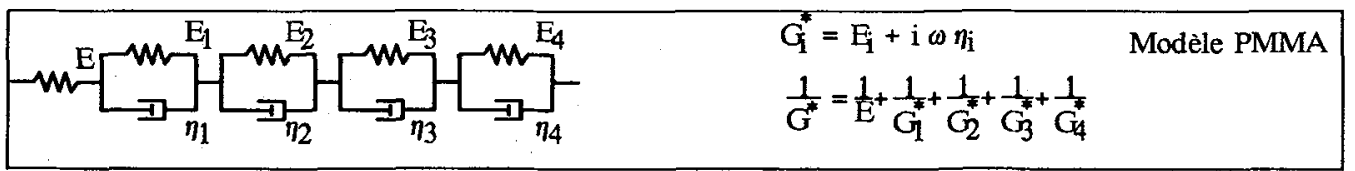

$C / C_{0}$ :vitesse de phase relative
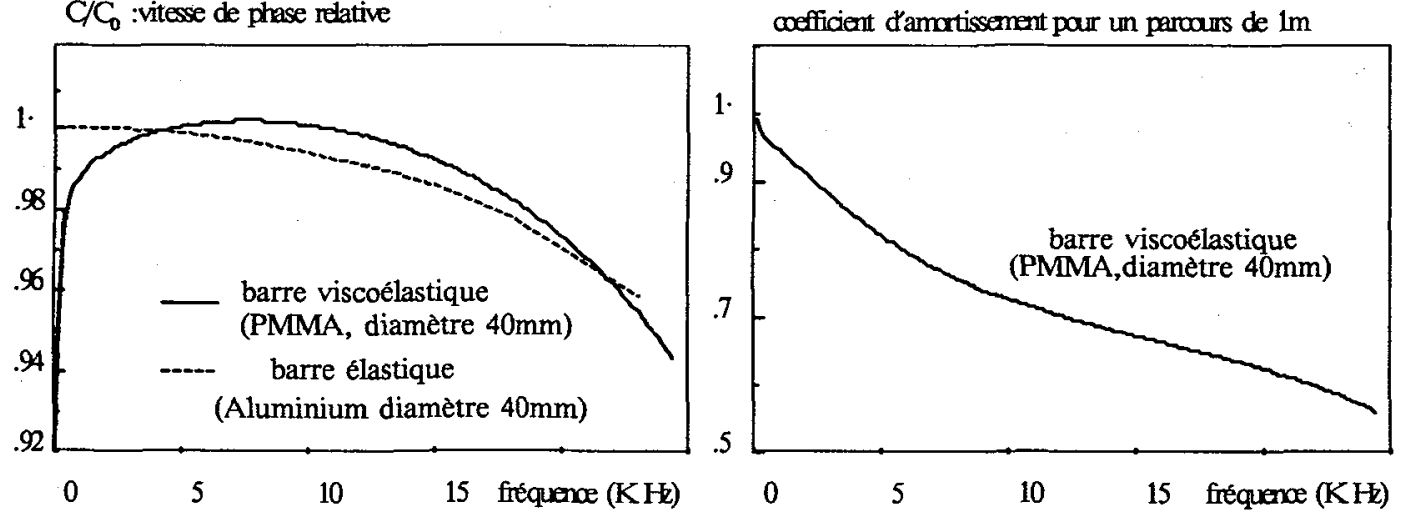

fig.5 Déphasage et amortissement, solution de l'équation de fréquence

Cette equation est résolue par une méthode de type Newton étendue au cas complexe $/ 17 /$, et nous donne les deux fonctions de la fréquence représentées sur la figure 5 . On décompose ensuite les signaux des jauges représentant les trois ondes en série de Fourier. On modifie ensuite les phases en tenant compte 
du déphasage da au transport (obtenu à partir de la relation phase-fréquence) et les amplitudes en tenant compte de l'amortissement (obtenu à partir de la relation amortissement-fréquence). On reconstruit ensuite les signaux par transformée de Fourier inverse. Cette méthode donne d'excellents résultats comme cela est illustré fig. 4..

\subsection{CAS DES BARRES ELASTIQUES}

Bien entendu, on retrouve les résultats de l'élasticité comme un cas particulier du modèle viscoélastique. Outre l'intérêt qu'il présente comme qualification de nos résultats, on remarquera que l'introduction d'une légère viscosité (en prenant un simple modèle de Zener) permet de mieux modéliser les effets réels de la dispersion observés sur une barre "élastique" après un long parcours, comme nous l'illustrons sur la fig. 6. Dans ce cas, l'apport de la viscosité reste toutefois marginal par rapport à l'apport de l'effet géométrique. Pour les parcours usuels dans les barres tels qu'on les rencontre dans les sytèmes SHPB élastiques, on peut done se contenter de la correction de dispersion elastique.

Deformation

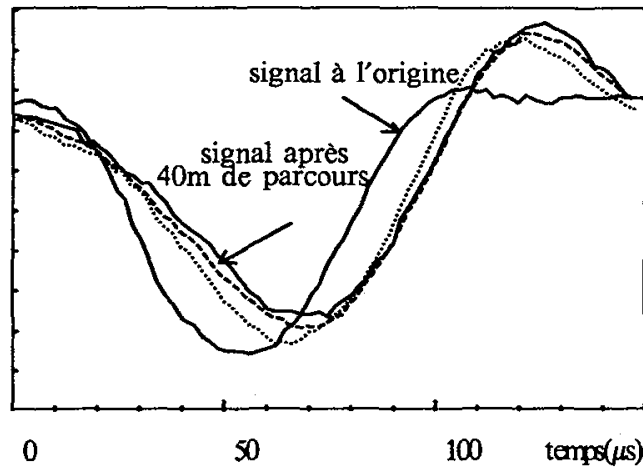

correction élastique

-..-.. correction viscoélastique

fig.6 Incidence de l'introduction d'une faible viscosité dans la correction de dispersion.

Cas d'un long parcours $(40 \mathrm{~m})$ dans une barre en aluminium

\subsection{CAS DES BARRES VISCOELASTIQUES}

Nous utilisons un modèle rhéologique unidimensionnel. Pour généraliser la loi au cas tridimensionnel, ce qui est bien entendu indispensable, nous supposons qu'il existe un coefficient de Poisson réel et constant, et donc facile à mesurer. Les coefficients de Lamé complexes se déduisent alors du module complexe unidimensionnel par des formules identiques à celles de l'élasticité. La détermination du module complexe $G^{*}$ sera donc suffisante pour calculer les parametres intervenant dans l'équation de fréquence. Ceci nous permet d'utiliser des modèles rhéologiques quelconques et donne par conséquent un caractère très général à notre approche. En particulier elle contient potentiellement les résultats établis par d'autres auteurs $/ 14,18,19,20,23,24,25,26 /$.

Pour le PMMA sur lequel nous travaillons, un simple modèle de Zener ne suffit pas. II est en particulier inapte à rendre correctement compte de la relation amortissement-fréquence obtenue expérimentalement. Nous avons donc dú identifier un modèle plus complexe composé d'un modèle de Zener avec trois modeles de Kelvin-Voigt en série. Il est présenté sur la figure 5 , où est explicitée la façon de calculer $G^{*}$.

\section{CONCLUSION}

Pour conclure, deux résultats d'essais réels sont présentés, sur les fig. $7 \mathrm{a}$ et $7 \mathrm{~b}$. Ils ont été obtenus et traités par le logiciel DAVID qui intègre toutes les techniques de calage et de correction présentées dans cet article. Ce logiciel est commercialisé. Le premier essai présenté est effectué sur un banc SHPB en acier avec une eprouvette en laiton. Le second est effectué sur un banc en PMMA avec une éprouvette en mousse de polyuréthane (utilisée dans l'industrie automobile).

On notera que, dans les deux cas, le comportement est correctement décrit, y compris pour les faibles déformations et que le module élastique trouvé est voisin du module calculé d'après le temps de transfert dans l'échantillon. Comme nous l'avons rappelé en introduction, nous n'avons pas abordé les aspects tridimensionnels de la réponse de l'échantillon. Nous devons cependant signaler qu'une "correction" de la relation contrainte-déformation basée sur les travaux de Malinowski et Klepaczko /7/ tenant compte des effets d'inertie et de frottement aux interfaces est inclue dans le logiciel DAVID. Son 
incidence est illustrée sur la figure $7 \mathrm{a}$.

On peut considérer que les résultats que nous avons présentés permettent d'optimiser le dépouillement de l'essai et d'accéder de façon précise aux forces et déplacements aux interfaces barres-échantillon et qu'en ce sens ils résolvent l'essentiel des problèmes inhérents au fonctionnement de la machine d'essai SHPB . En conséquence, pour les essais de comportement, ils permettent d'observer la zone des faibles déformations de l'ordre des $10^{-3}$, ce qui permet d'étudier, avec la méthode SHPB, le comportement des matériaux géotechniques.

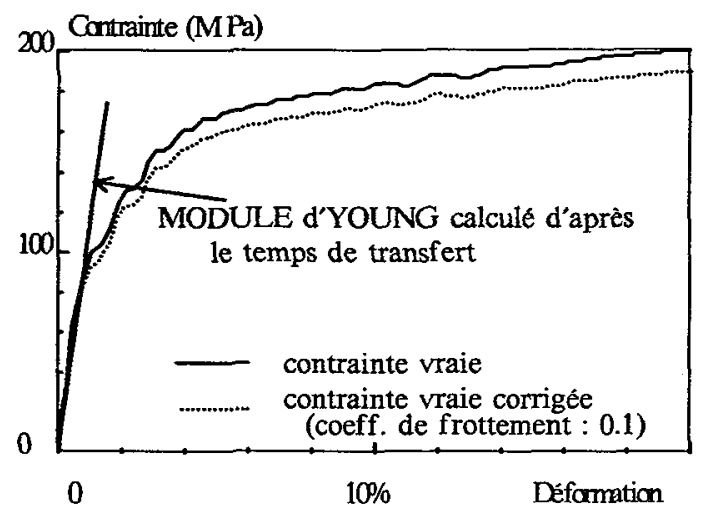

fig.7a Essai sur un echantillon de LAITON, vitesse de deformation moyenne $(600 / \mathrm{s})$

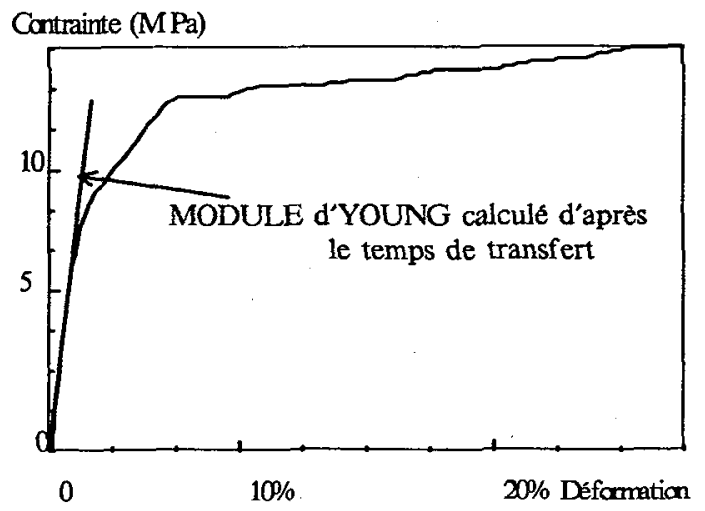

fig.7b Essai sur MOUSSE POLYURETHANE, vitesse de déformation moyenne $(800 / \mathrm{s})$

\section{REFERENCES}

/ $1 /$ Kolsky H., Proc. Phys. Soc., B62 (1949), 676

/ 2 L Lindholm, U.S., J. Mech. Phys. Solids, 12 (1964), 317

/ 3/ Bertholf, L.D. and Karnes, J. Mech. Phys. Solids, 23 (1975), 1

/ 4/ Davies, R.M., Phil. Trans., A240 (1948), 375

/ $5 /$ Davies, E.D.H., and Hunter, S.C., J. Mech. Phys. Solids, 11 (1963), 155

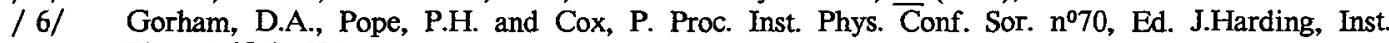
Phys. (1984), 381

/ 7/ Malinowski, J.Z. and Klepaczko, J.R. , Int. J. Mech. Sci., 28 (1986), 381

/ 8/ Sokolovsky, Prikl. Mat. Mekh., 12 (1948), 261

/ 9/ Malvern, L.E., J.Appl. Mech., 18 (1951), 203

/10/ Gary, G. et Klepaczko, J.R., Logiciel de dépouillement de l'essai de compression par barres de Hopkinson (DAVID), Paris/Metz (1988).

/11/ Pochhammer, L., J. für die Reine und Angevande Mathematic, 81 (1876), 324.

/12/ Chree, C., Cambridge Phil. Soc., Trans. 14 (1889), 250

/13/ Kolsky, H., Stress Waves in Solids, Clarendon Press, Oxford (1953)

/14/ Kolsky, H., Phil. Mag., 1 (1956), 693.

/15/ Coquin, G.A., J. ASA, $\underline{36}$ (1964), 1074

/16/ Klepaczko, J.R. et Gary, G., Greco Géomatêriaux (CNRS), Rapport 1990

/17/ Zhao, $\mathrm{H}$. , Thèse de Doctorat, ENPC et Ecole Polytechnique, à paraitre.

/18/ Riecker, M., Bull. Seism. Soc. Amer., 33 (1947), 197

/19/ Nolle, A.W., J. ASA, 19 (1947), 194

/20/ Hillier, K.W., Proc. Phys., Ser B, 2 (1949), 701

/21/ Berrj, D.S. and Hunter, S.C., J. Mech. Phys. Solids, 4 (1956), 2

/22/ Bland, D.R. and Lee, E.H., J. Appl. Mech., 3 (1956), 23

/23/ Bland, D.R., The Theory of Linear Viscoelasticity, Oxford Univ. Press., Oxford (1960)

/24/ Follansbee, P.S. and Franz, C., J. 105 Engng. Mater. and Tech., 105 (1983), 61

/25/ Morris, D.R. and Watson, A.J., Proc. Inst. Phys. Conf. Ser. no102, Inst. phys. (1990), 519

/26/ Gong, J.C., Malvern, L.E. and Jenkins, D.A., J. Engng. Mater. and Tech., 112 (1990), 309 